\title{
Biofilm Formation in UPEC Isolates and its Association with Extended-Spectrum $\beta$-Lactamase Production and MDR in Hospitalized Patients: Gurugram, India
}

\author{
Rishabh Rajput $^{{ }^{*}}$, Surendra Sarsaiya ${ }^{2,3}$, Naresh Kumar ${ }^{4}$ and Swati Shekhawat ${ }^{5}$
}

${ }^{1}$ Department of Microbiology \& Molecular Biology, Modern Diagnostic \& Research Centre, Gurugram, Haryana - India

${ }^{2}$ Laboratory of Basic Pharmacology \& Joint International Research Laboratory of

Ethnomedicine of Ministry of Education, Zunyi Medical University, Zunyi, Guizhou, China

${ }^{3}$ CES Analytical \& Research Services India Pvt. Ltd, Bhopal-Madhya Pradesh-India

${ }^{4}$ Department of Medical Laboratory Sciences, Lovely Professional University, Punjab

${ }^{5}$ Research Scholar, Dr. A.P.J. Abdul Kalam University, Indore, India

*Corresponding author

\section{A B S T R A C T}

\section{Keywords}

Uropathogenic E.coli, UTI, Multidrug resistance, Extended-Spectrum $\beta$-lactamase, ESBL, E.coli, CLSI, ATCC, Hospitalized

\section{Article Info}

Accepted:

07 January 2021

Available Online:

10 February 2021
Urinary tract infections (UTI) are perhaps the most regular issues in hospitalized patients. Gram negative bacteria which produces Biofilm and Extended-range B-lactamases (ESBL) have become the serious issue in the medical clinics across the globe. Increase in biofilm \& ESBL forming organisms in recent years has led to the limitation of treatment option. This study was done to evaluate the Biofilm production in uropathogenic E. coli (UPEC) and its association with ESBL, its antibiogram and Multi-drug resistance (MDR) status in hospitalized patients. 885 urine samples (non-repetitive) were collected in the study. A total of $106(11.98 \%)$ UPEC strains were isolated. Females were found to be more infected as compare to males. The most affected age group was $>46$ years. $73.58 \%$ of the UPEC isolates were Biofilm producer (BFP). MDR was found in $84.91 \%$ strains (Biofilm +VE: $97.44 \%$; Biofilm -VE" 50.0\%). Tissue culture plate (TCP) method was most sensitive test method for the detection of biofilms. The most sensitive drug in BFP's were Imipenem $(82.0 \%)$, Ertapenem (81.0\%), Nitrofurantoin (72.0\%) and Amikacin (69.0\%) and Nitrofurantoin, Imipenem (100.0\% each), Amikacin (96.0\%) and Ertapenem, Piperacillin/Tazobactam (93.0\% each) in Biofilm non producers. The ability of biofilm production was found significantly higher in ESBL positive UPEC strains. An association was found between biofilm production and antibiotic resistance. Therefore, the UTIs caused by BFP's E.coli may promote the colonization and increased the incidence rate of UTIs. It was found that biofilm makes the organism more resistant to the antibiotics and virulent as compare to non-biofilm producers (NBFP's). The UPEC employ their biofilm forming abilities to invade and successfully occupy tissues in the urogenital tract. 


\section{Introduction}

UTI is the most frequent problems common in patients admitted to the hospital and caused by Escherichia coli which accounts for $90.0 \%$ of community-acquired and $50.0 \%$ of hospital-acquired $\mathrm{UTI}^{1,2}$. Biofilm formation is an important virulence factor in many nosocomial infections so that in $65 \%$ of the nosocomial infections biofilm are formed ${ }^{3}$. It has been recommended that some chromosomal gene re-arrangement occurs upon acquisition of the ESBL plasmid. It is possible that higher mortality and severity of infection caused by ESBL producing isolates is due the expression of several virulence genes simultaneously, rather than gaining new virulence genes ${ }^{4}$.

It has been discovered that biofilm made the microcolonies impermeable to anti-microbials and bound the agents at the external surface of the matrix layer. The presence of other factors in UPEC further muddle the circumstance, by shielding these isolates from the activity of anti-microbials, and made the treatment of UTIs complex by the development of resistance against antibiotics ${ }^{5}$.

The prevalence of MDR in BFP's UPEC has been reported ${ }^{6}$ more in comparison to NBFP's isolates. MDR could be due to the spread of hospital strains of UPEC, and the high prevalence of ESBL (88\%), AmpC (22\%) and MBL (6\%) positive isolates is possibly due to the creation of selective drug pressure because of frequent use of cephalosporins and other anti-mircobials 5 .

BFP E. coli were recalcitrant to immune factors and antibiotic therapy and were frequently responsible for chronic UTIs. Usually, floating cells are entirely eradicate at the antibiotic levels predicted by minimuminhibitory-concentration (MIC) study. Therefore, efficient control will need an rigorous effort to build up newer therapeutic agent that seek to prevent the creation of biofilm or encourage the biofilm detachment ${ }^{5}$. The antibiotic sensitivity patterns have changed over time, but the spectrum of agents causing urinary tract infections has remained relatively constant, with E.coli being the most common isolate ${ }^{7}$.

Many bacteria are proficient in forming biofilms. It is defined as matrix-enclosed microbial residents which are adherent to each other. The biofilms develop on surfaces can be regarded as a universal strategy of bacterial for its survival and optimum positioning to efficiently use available nutrients. The gel-like state, mostly consisting of polysaccharides, prevent the access of antibacterial agents, such as antibodies, white blood cells and antibiotics, so that sessile bacterial cells in biofilms can withstand host immune responses and are much less prone to antibiotics than in their non-attached individual planktonic state ${ }^{8-10}$. Due to differential genes expressions in the biofilms, the microorganisms changed phenotypically as they attach to the surfaces ${ }^{11}$.

\section{Materials and Methods}

\section{Study design and area}

This prospective study was conducted from urine samples collected from hospitalized patients complaining about UTIs. The samples were processed in Bacteriology Section, Department of Microbiology, Modern Diagnostic \& Research Centre, Gurugram, Haryana-India.

Inclusion and exclusion criteria: All the UPEC having pure growth were included in the present study. Insignificant bacteriuria, mixed growth and isolates other than E.coli were excluded. 


\section{Processing of the samples}

All samples were processed within 1-2 hours of the collection, and in case of delay, the specimens were refrigerated at $4^{\circ} \mathrm{C}$. All urine samples were cultured by the semiquantitative method. In short, $0.01 \mathrm{ml}$ of urine was inoculated on Cysteine lactose electrolyte deficient agar (CLED Agar) (Hi-media Pvt. Ltd) by crisscross streaking using disposable calibrated flexi-loop (Hi-media Pvt. Ltd) and incubated for $18-24$ hours at $37^{\circ} \mathrm{C}$ under aerobic conditions. A pure growth of the gram-negative isolate on a colony count $\geq 10^{5}$ colony forming units was considered as significant bacteriuria. Isolation and identification of the strains were done following their morphology in Gram's staining, culture characteristics and biochemical properties. Plates with no growth were incubated for another 24 hours before interpreting it as a negative culture.

\section{Quality control}

The bacterial suspension was prepared and was adjusted to a $0.5 \mathrm{McFarland}$ standard solution (Hi-media Pvt. Ltd). American Type Culture Collection (ATCC) standard reference strains P.aeruginosa ATCC-27853, S.aureus ATCC-25923, E.coli ATCC-25922 were used as a quality control strains for antimicrobial susceptibility testing. All the ATCC strains used in the current prospective study were procured from Microbiologics, USA.

\section{Antibiotic sensitivity testing}

Antibiotic sensitivity testing was done by Kirby-Bauer disc diffusion method on Mueller-Hinton agar, and interpretation of the results was done as described by CLSI guidelines with commercially available antibiotics (Hi-Media Lab Pvt. Ltd) ${ }^{12}$. Antibiotics discs used (drug concentration in $\mu \mathrm{g})$ were Ampicillin (AMP)-10, Gentamicin (GEN)-10, Tobramycin (TOB)-10, Amikacin (AK)-10, Amoxicillin-Clavulanic Acid (AMC)-20/10, Ampicillin/Sulbactum (A/S)20/10, Piperacillin/Tazobactum (P/T)-100/10, Cefuroxime (CXM)-30, Cefepime (CPM)-30, Ceftazidime (CAZ)-30, Aztreonam (AT)-15, Cefoxitin (CX)-30, Levofloxacin (LE)-5, Ciprofloxacin (CIP)-5, Imipenem (IMP)-10, Ertapenem (ETP)-10, Cotrimoxazole (COT)25, Tetracycline (TET)-30, Nitrofurantoin (NIT)-300.

\section{Detection of ESBL production}

All the E.coli isolates were subjected for the detection of ESBL production. The Screening and phenotypic confirmation of the strains was performed as per the CLSI guidelines ${ }^{12}$. For Quality control of the ESBL test, K.pneumoniae (ATCC-700603) was used as a positive control, and E.coli (ATCC-25922) as a negative control.

\section{Statistical analysis}

Chi-square test was used for statistical analysis of the data. A $p$-Value of $<0.05$ was considered as statistical significant.

\section{Detection of biofilm production by E.coli}

Biofilm detection in E.coli was done by three different methods: Tube method (TM), Congo Red Agar method (CRA) and Tissue culture plate method (TCP).

\section{Tube method}

E.coli was inoculated in $10 \mathrm{ml}$ of trypticase soy broth (TSB) with $1 \%$ glucose in test tubes. Incubation was done at 37 degree $C$ for 24 hours. After incubation, tubes were decanted and washed off with phosphate buffer saline (PBS) with $\mathrm{pH}=7.3$ and dried. Crystal violet $(0.10 \%)$ was used to stain 
Tubes. Excess stain was removed with deionized water. Tubes were dried in an upturned place. According to the results of control strains, the scoring was done. When a visible film lined the wall and the bottom of the tube, biofilm formation was considered positive. The amount of biofilm produced was scored as 1:weak/none, 2: moderate and 3: high/strong. The experiment was repeated three times and performed in triplicate ${ }^{13}$.

\section{Congo red agar method}

CRA medium was prepared with brain heart infusion (BHI) agar and Congo red indicator (Hi-media India Pvt. Ltd.). Firstly, Congo red stain was prepared as a concentrated aqueous solution and autoclaved at $121^{\circ} \mathrm{C}$ for 15 minutes. Then it was added to the autoclaved $\mathrm{BHI}$ agar having sucrose at $55^{\circ} \mathrm{C}$. CRA plates were inoculated with test organisms and incubated at $37^{\circ} \mathrm{C}$ for 24 hours aerobically. Black colonies with a dry crystalline consistency were considered as BFP's and red colored colonies were considered as BFNP's. The test was performed and repeat three times ${ }^{14}$.

\section{Tissue culture plate method}

TCP method is the quantitative test and is considered the gold-standard method for biofilm detection ${ }^{15}$. Isolates wase inoculated in $10 \mathrm{ml}$ of trypticase soy broth (TSB) with $1 \%$ glucose.

$24 \mathrm{hrs}$ incubation at $37^{\circ} \mathrm{C}$. Dilution of the culture was done with fresh medium (1:100). Individual wells of sterile 96 well-flat bottom polystyrene tissue culture treated plates were filled with $200 \mu \mathrm{l}$ of the diluted cultures with fresh medium. The same process was used for the control organisms. Negative control wells were inoculated with sterile broth. Incubation was done for $24 \mathrm{hrs}$ at $37^{\circ} \mathrm{C}$. The content of each well was removed by gentle tapping after incubation.0.2 $\mathrm{ml}$ of PBS was used to wash the wells $(4 \mathrm{x})$. This removed freefloating bacteria. Biofilm formed by bacteria adherent to the wells were fixed by $2 \%$ sodium acetate and stained by crystal violet $(0.1 \%)$. Excess stain was removed with deionised water and dried. Optical density (OD) was obtained by using micro ELISA auto reader (Benesphera) at the wavelength of $570 \mathrm{~nm}$. The experiment was performed in triplicate and was repeated three times. The interpretation of biofilm production was made according to the criteria of Stepanovic et al., $2007^{16}$ (Table-1).

\section{Results and Discussion}

In the current study, 885 urine samples suspected of urinary tract infection were collected from hospitalized patients in and around Gurugram, Haryana. The median age was of 54 years (age range: 0-92 years). There were $66.89 \% \quad(592 / 885) \quad$ culture-negative samples, with the median age of 52 years and age range of 0-92 years. In the present study, $11.98 \% \quad(106 / 885)$ samples were found positive for E.coli with the median age of 58 years (age range: 0-87 years). Prevalence of urinary tract infection due to E.coli among the age wise distribution had shown no significant difference $(p=0.155)$ (Table-2). There were $52.43 \%$ (464/885) males included in this study and $79.74 \%$ (370/464) individuals presented with the negative urine culture. Rest of the $6.68 \%$ (31/464) males were presented with the positive urine culture for E.coli and others were 13.58 (63/464). There were $47.57 \% \quad(421 / 885)$ females included in the study and $52.73 \%$ (222/421) individuals presented with the negative urine culture. Rest of the $17.81 \%$ (75/421) females shown with the positive urine culture for E.coli and others were 29.45 (129/421). Prevalence of urinary tract infection among the different gender showed a significant difference $(p=0.000)$ (Table-2). 


\section{Identification of biofilm formation in E.coli}

Biofilm formation allows the strains to persist a long time in the genitourinary tract and interfere with bacterial eradication. In the current study, $73.58 \%$ of isolates were invitro positive by TCP method, $58.49 \%$ strains by Tube method and $23.58 \%$ strains by Congo red agar method for Biofilm production. The current study included 106 E.coli strains for the detection of Biofilm formation. In TM, there were $13(12.26 \%)$ isolates showed strong positive, 44 strains $(41.51 \%)$ were moderately positive, 05 strains $(4.72 \%)$ were weakly positive and rest 44 strains $(41.51 \%)$ were negative. The results are shown in Table-3. In CRA method, 11 isolates $(10.38 \%)$ showed strong positive, 14 isolates $(13.21 \%)$ were weakly positive, 81 isolates $(76.42 \%)$ were negative. The results are shown in Table-4. In TCP method, 43 strains $(40.57 \%)$ showed strong positive, 35 isolates $(33.02 \%)$ were moderately positive and remained 28 strains $(26.42 \%)$ were negative. The results are shown in Table-5. OD value of stained adherent Biofilm was obtained with a micro ELISA reader at wavelength $490 \mathrm{~nm}$. The OD value less than 0.102 was considered as non-Biofilm producers, $0.102-0.240$ as moderate Biofilm producer and more than 0.240 as strong Biofilm producer (Table-5).

\section{Sensitivity and specificity of different methods}

Three different methods were evaluated for the identification of the Biofilm production. Tissue culture plate method was found to be the best compared to the others. The sensitivity and specificity of all the three methods were evaluated and shown in Table6.

TCP method was found to be more sensitive as compared to TM and CRA method. The further analysis of the Biofilm producing and non-producing E.coli was done, and antibiotics sensitivity tests were performed.

\section{Antibiotic sensitivity pattern of biofilm \& non-biofilm producing E.coli in hospitalized patients}

In the present study, total 106 E.coli were isolated, and 78 were found to be Biofilm producer by TCP method in Hospitalized patients (Table 7).

In Biofilm positive E.coli $(\mathrm{n}=78)$ isolated from hospitalized patients, the most sensitive drugs were Imipenem (82.0\%), Ertapenem (81.0\%), Nitrofurantoin (72.0\%), Amikacin (69.0\%). Resistant to Ampicillin (99.0\%), Ciprofloxacin (97.0\%), Levofloxacin (95.0\%), Cefuroxime (88.0\%) (Fig. 1).

In Biofilm negative E.coli $(\mathrm{n}=28)$ isolated from hospitalized patients, the most sensitive drugs were Imipenem, Nitrofurantoin (100.0\% each), Amikacin (96.0\%), Ertapenem, Piperacillin/Tazobactam (93.0\% each), Amoxicillin/clavulanic acid(89.0\%). On the other hand resistant to Levofloxacin (64.0\%), Ciprofloxacin (61.0\%), Ampicillin (46.0\%), Tetracycline, Cotrimoxazole (43.0\% each) (Fig. 1).

\section{Antibiotic sensitivity pattern of biofilm \& non-biofilm producing E.coli with reference to ESBL}

It was crucial understanding the sensitivity and resistant pattern of Biofilm and nonBiofilm producing E.coli in both Hospitalized and Non-Hospitalized patients concerning ESBL as it might be helpful to determine the antimicrobial therapy. In the following data, it is evident that sensitivity pattern changes in hospitalized and non-hospitalized patients concerning ESBL in Biofilm and non-Biofilm producing E.coli. Hence the present study 
looked through the several categories described below.

In ESBL +VE, biofilm +VE E.coli $(\mathrm{N}=34)$, the sensitivity pattern was Imipenem, Ertapenem (97.0\% each), Nitrofurantoin (88.0\%), Amikacin (82.0\%), Cefuroxime $(79.0 \%)$ and resistant to Ciprofloxacin (100.0\%), Levofloxacin (97.0\%), Ampicillin/ Sulbactam (85.0\%), Tetracycline, Tobramycin, Cotrimoxazole (79.0\% each). In ESBL +VE, biofilm -VE E.COLI $(\mathrm{N}=4)$, the sensitivity pattern was Imipenem, Nitrofurantoin (100\% each), Amikacin, Amoxicillin/clavulanic acid, Cefoxitin, Ertapenem, Piperacillin/Tazobactam $\mathbf{7 5 . 0 \%}$ each), Ampicillin/Sulbactam, Ciprofloxacin, Cotrimoxazole $(25.0 \%$ each $)$ and resistant pattern was Levofloxacin, Tetracycline, Tobramycin (100.0\% each), Ciprofloxacin, Cotrimoxazole $\quad(75.0 \% \quad$ each $)$, Ampicillin/Sulbactam, Gentamicin $\quad(50.0 \%$ each).

In ESBL -VE, biofilm +VE E.coli $(\mathrm{N}=44)$, the sensitivity pattern was Imipenem (70.0\%), Ertapenem (68.0\%), Amikacin, Nitrofurantoin (59.0\% each), Cefoxitin, Tetracycline (36.0\% each) and resistant pattern was Ampicillin (98.0\%), Ciprofloxacin (95.0\%), Levofloxacin (93.0\%), Ampicillin/Sulbactam $(89.0 \%)$

In ESBL -VE, biofilm -VE E.coli N=24, the sensitivity pattern was Amikacin, Imipenem, Nitrofurantoin (100.0\% each), Piperacillin/ Tazobactam, Ertapenem (96.0\% each), Amoxicillin/clavulanic acid, Aztreonam, Cefepime, Ceftazidime $(92.0 \%$ each), Tobramycin $(88.0 \%)$ and resistant pattern was Ciprofloxacin, Levofloxacin (58.0\% each), Ampicillin, Cotrimoxazole $(38.0 \%$ each $)$, Tetracycline (33.0\%), Cefuroxime (25.0\%).

\section{Correlation of biofilm producing strains with MDR strains}

The strain exhibited resistance to various commonly used antibiotics compared to the strains producing biofilm. It was found that the resistance patterns differ depending upon the strains of E.coli producing Biofilm. There was a significant correlation between Biofilm production and resistance to multiple classes of antibiotics. Biofilm producing E.coli were much MDR than the Non-Biofilm producing E.coli. In the current study, among 106 E.coli, MDR were $84.94 \%$ (90/106), and non-MDR were $15.09 \%$ (16/106). In the case of Biofilm positive UPEC $73.58 \%$ (78/106), MDR were $97.44 \%$ (76/78), and non-MDR were $2.56 \%$ (2/78) whereas $50.00 \%(14 / 28)$ were MDR and $50.00 \%(14 / 28)$ were non-MDR in Biofilm negative UPEC $26.42 \%$ (28/106). In this case, biofilm producing E.coli showed higher MDR than the Biofilm non producing E.coli. (Table-8) The antimicrobial classes are depicted in the Table-9.

Table.1 Interpretation of Biofilm Production by TCP method

\begin{tabular}{|c|c|}
\hline Average OD Value & Biofilm Production \\
\hline$\leq$ Odc $/$ Odc $<\sim \leq \mathbf{2 x}$ ODc & Non/Weak \\
\hline $\mathbf{2 x}$ ODC $<\sim \leq \mathbf{4 x}$ ODc & Moderate \\
\hline$>\mathbf{4 x}$ ODc & Strong \\
\hline
\end{tabular}

Optical density cut-off value $(\mathrm{ODc})=$ average $\mathrm{OD}$ of negative control $+3 \mathrm{x}$ standard deviation $(\mathrm{SD})$ of negative control 
Table.2 Demographical features of the studied samples

\begin{tabular}{|c|c|c|c|c|c|}
\hline Features & TOTAL (n) & $\operatorname{Neg}(\%)$ & E.coli $(\%)$ & Others & $p$-Value \\
\hline & 885 & $592(66.89)$ & $106(11.98)$ & $187(21.13)$ & \multirow[t]{2}{*}{ NA } \\
\hline MEDIAN AGE (RANGE) & $54(0-92)$ & $52(0-92)$ & $58(0-87)$ & $57(0-88)$ & \\
\hline \multicolumn{6}{|l|}{ AGE GROUP } \\
\hline $0-15$ & $77(8.70)$ & $50(64.94)$ & $10(12.99)$ & \multirow[t]{5}{*}{ NA } & \multirow[t]{5}{*}{0.155} \\
\hline $16-30$ & $135(15.25)$ & $106(78.52)$ & $13(9.63)$ & & \\
\hline $31-45$ & $135(15.25)$ & $97(71.85)$ & $10(7.41)$ & & \\
\hline $46-60$ & $201(22.71)$ & $129(64.18)$ & $28(13.93)$ & & \\
\hline$>60$ & $337(38.08)$ & $210(62.31)$ & $45(13.35)$ & & \\
\hline \multicolumn{6}{|l|}{ GENDER } \\
\hline MALE & $464(52.43)$ & $370(79.74)$ & $31(6.68)$ & \multirow[t]{2}{*}{ NA } & \multirow[t]{2}{*}{0.000} \\
\hline FEMALE & $421(47.57)$ & $222(52.73)$ & $75(17.81)$ & & \\
\hline
\end{tabular}

Table.3 Biofilm producing E.coli by tube method ( $\mathrm{n}=106)$

\begin{tabular}{|c|c|c|c|}
\hline S.NO & Observation & Result & Prevalence (\%) \\
\hline $\mathbf{1}$ & +++ & 13 & 12.26 \\
\hline $\mathbf{2}$ & ++ & 44 & 41.51 \\
\hline $\mathbf{3}$ & + & 5 & 4.72 \\
\hline $\mathbf{4}$ & - & 44 & 41.51 \\
\hline \multicolumn{2}{|c|}{ Total } & 106 & 100.00 \\
\hline
\end{tabular}

Table.4 Biofilm producing E.coli by congo red method (n=106)

\begin{tabular}{|c|c|c|c|}
\hline S.NO & Observation & Result & $\begin{array}{c}\text { Prevalence } \\
(\mathbf{\%})\end{array}$ \\
\hline $\mathbf{1}$ & ++ & 11 & 10.38 \\
\hline $\mathbf{2}$ & + & 14 & 13.21 \\
\hline $\mathbf{3}$ & - & 81 & 76.42 \\
\hline & Total & 106 & 100.00 \\
\hline
\end{tabular}

'++' - Positive, '+' - Weekly Positive, '-' - Negative

Table.5 Classification of Uropathogenic E.coli based on O.D value obtained by tissue culture plate method

\begin{tabular}{|l|c|c|c|c|}
\hline $\begin{array}{l}\text { Mean O.D } \\
\text { Value }\end{array}$ & Adherence & $\begin{array}{c}\text { Biofilm } \\
\text { Formation }\end{array}$ & Result & $\begin{array}{c}\text { Prevalence } \\
(\mathbf{\%})\end{array}$ \\
\hline $\mathbf{< 0 . 1 0 2}$ & Non & Non/Weak & 28 & 26.42 \\
\hline $\mathbf{0 . 1 0 2} \mathbf{- 0 . 2 4 0}$ & Moderate & Moderate & 35 & 33.02 \\
\hline$>\mathbf{0 . 2 4 0}$ & Strong & Strong & 43 & 40.57 \\
\hline & Total & & 106 & 100.00 \\
\hline
\end{tabular}


Table.6 Test sensitivity \& specificity of different screening methods of biofilm formation in E.coli

\begin{tabular}{|l|c|c|}
\hline METHOD & $\begin{array}{c}\text { SENSITIVITY } \\
(\boldsymbol{\%})\end{array}$ & $\begin{array}{c}\text { SPECIFICITY } \\
(\boldsymbol{\%})\end{array}$ \\
\hline TCP & 73.58 & 26.42 \\
\hline TUBE & 58.49 & 41.51 \\
\hline CRA & 23.58 & 76.42 \\
\hline
\end{tabular}

Table.7 Detailed analysis of biofilm production in E.coli by TCP method $(\mathrm{n}=106)$

\begin{tabular}{|l|c|c|c|c|c|}
\hline \multicolumn{1}{|c|}{ TCP Method } & Total & $\begin{array}{c}\text { Biofilm } \\
\text { Positive }\end{array}$ & $\begin{array}{c}\text { Biofilm } \\
\text { Negative }\end{array}$ & Positive (\%) & $\begin{array}{c}\text { Negative } \\
(\boldsymbol{\%})\end{array}$ \\
\hline Total E.coli & 106 & 78 & 28 & $\mathbf{7 3 . 5 8}$ & $\mathbf{2 6 . 4 2}$ \\
\hline ESBL +ve Hospitalized & 38 & 34 & 4 & $\mathbf{8 9 . 4 7}$ & $\mathbf{1 0 . 5 3}$ \\
\hline ESBL -ve Hospitalized & 68 & 44 & 24 & $\mathbf{6 4 . 7 1}$ & $\mathbf{3 5 . 2 9}$ \\
\hline
\end{tabular}

Table.8 Detailed analysis of MDR production

\begin{tabular}{|l|c|c|c|}
\hline \multicolumn{1}{|c|}{ TCP Method } & Total & MDR (\%) & $\begin{array}{c}\text { Non MDR } \\
(\%)\end{array}$ \\
\hline Total E.coli & 106 & $90(84.91)$ & $16(15.09)$ \\
\hline Biofilm Positive & 78 & $76(97.44)$ & $2(2.56)$ \\
\hline Biofilm Negative & 28 & $14(50.0)$ & $14(50.0)$ \\
\hline
\end{tabular}

Table.9 MDR pattern of biofilm producing and Biofilm non producing E.coli

\begin{tabular}{|c|c|c|c|c|c|c|}
\hline S.No & $\begin{array}{c}\text { Antimicrobial } \\
\text { resistant } \\
\text { Class }\end{array}$ & $\begin{array}{c}\text { Total } \\
\text { E.coli }\end{array}$ & $\begin{array}{c}\text { Biofilm } \\
\text { Positive } \\
\text { E.coli } \mathbf{( n = 7 8 )}\end{array}$ & $\begin{array}{c}\text { Biofilm } \\
\text { Positive } \\
\text { E.coli } \%\end{array}$ & $\begin{array}{c}\text { Biofilm } \\
\text { Negative } \\
\text { E.coli }(\mathbf{n}=\mathbf{2 8})\end{array}$ & $\begin{array}{c}\text { Biofilm } \\
\text { Negative } \\
\text { E.coli } \%\end{array}$ \\
\hline $\mathbf{1}$ & 10 Classes & 7 & 7 & 9 & 0 & 0 \\
\hline $\mathbf{2}$ & 9 Classes & 7 & 7 & 9 & 0 & 0 \\
\hline $\mathbf{3}$ & 8 Classes & 31 & 30 & 38 & 1 & 4 \\
\hline $\mathbf{4}$ & 7 Classes & 21 & 17 & 22 & 4 & 14 \\
\hline $\mathbf{5}$ & 6 Classes & 5 & 5 & 6 & 0 & 0 \\
\hline $\mathbf{6}$ & 5 Classes & 8 & 4 & 5 & 4 & 14 \\
\hline $\mathbf{7}$ & 4 Classes & 6 & 3 & 4 & 3 & 11 \\
\hline $\mathbf{8}$ & 3 Classes & 5 & 3 & 4 & 2 & 7 \\
\hline $\mathbf{9}$ & 2 Classes & 7 & 1 & 1 & 6 & 21 \\
\hline $\mathbf{1 0}$ & 1 Classes & 1 & 0 & 0 & 1 & 4 \\
\hline $\mathbf{1 1}$ & 0 Classes & 8 & 1 & 1 & 7 & 25 \\
\hline & & 106 & 78 & 100 & 28 & 100 \\
\hline
\end{tabular}


Fig.1 Antibiogram of biofilm positive and negative isolates of E.coli

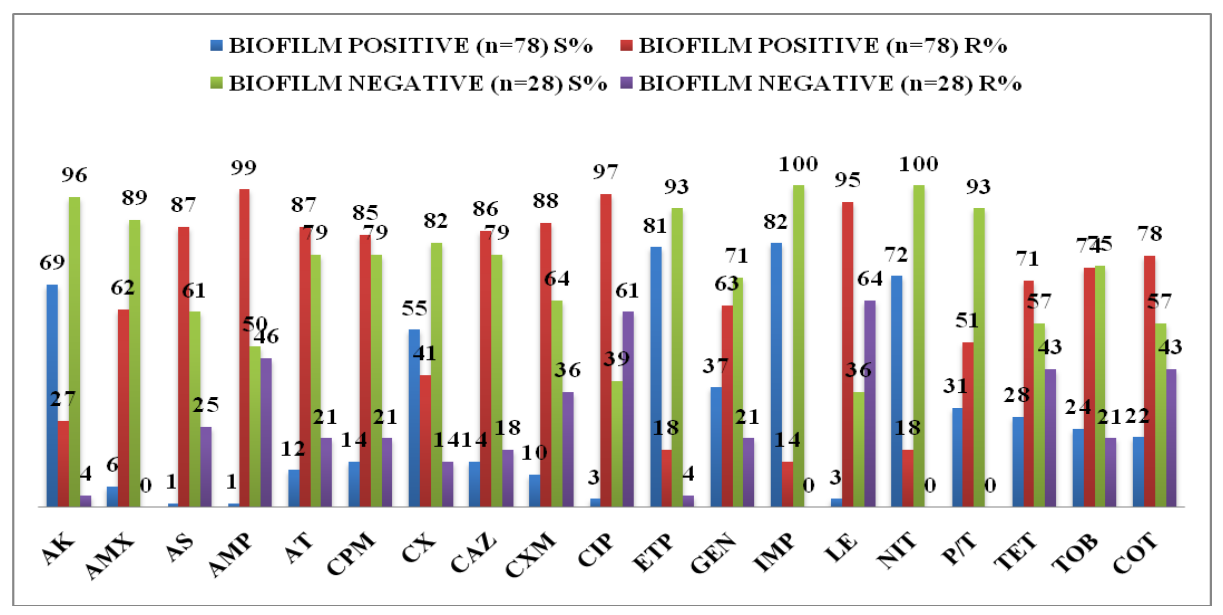

The present study evaluated the Biofilm formation in UPEC isolates and its association with ESBL production and MDR from the hospitalized patients from in and around Gurugram, Haryana. To the best of our knowledge, this is the first research from our area with an exclusive focus on biofilm production in the most predominate uropathogen: Escherichia coli.

In the current study, the total number of UPEC isolated from hospitalized patients were $36.18 \%$ whereas other similar studies reported different isolation rates Jigna et al., $(60.0 \%)^{17}$, Poovendran et al., $(62.96 \%)^{18}$, Niveditha et al., $(70.0 \%)^{19}$, Shahidul et al., $(75.0 \%)^{20}$. The variation observed in the prevalence of the UPEC was may be due to the different area of study. E.coli is the most frequent uropathogen. Acute UTI caused by UPEC can lead to recurrent infection, which can be defined as either re-infection or relapse $^{21} \cdot 52.43 \%$ were males and $47.57 \%$ were females included in this study, among them E.coli was $6.68 \%$ and $17.81 \%$ respectively. The prevalence of infection by E.coli was more in females as compare to males (pValue- 0.000). Similar findings were observed in the study done at Annamalai Nagar, India ${ }^{18}$ and Dhaka, Bangladesh ${ }^{20}$.
46-60 years age group was found to be more infected by E.coli followed by $>60,0-15,16-$ $30,31-45$ age group. No statistical significant difference was found among different age groups. Therefore, the infection of UPEC is common in all age groups whereas in the other study done higher isolation rate was observed in 51-60 $\mathrm{yrs}^{18}$. A similar results were found in the study done by Niveditha $e t$ al who found UTI in the elderly patients who were aged $>60$ years ${ }^{19}$. Similar findings had been reported among the non instutionalized elderly populations, in which genitourinary infections were the second most common form of the infections, accounting for nearly $25 \%$ of the identified infections ${ }^{22}$. This recommended that the elder who reside in the long-term-care facilities had higher prevalence of the chronic genitor-urinary symptoms and bacteriuria, and that they were at a risk for urinary catheter associated infections.

A biofilm is the thin layer of the microorganisms that adhere to the surface of an inorganic or organic structure, together with their polymers. These are the predominant phenotype of nearly all the bacteria in their natural habitats, whether they are pathogenic or environmental. 
In the present study, three different methods were carried out for the detection of biofilm formation. TCP method was found to be more superior as compare to CRA and TM (Test Sensitivity $=$ TCP:73.58\%; TM: 58.49\%; CRA: 23.58\%). So TCP method was considered as gold standard. Similar kind of findings are observed in study done by Hassan et $a l .{ }^{23}$ and Golia et al. ${ }^{24}$

In the current study, $73.58 \%$ UPEC were positive and $26.42 \%$ were negative invitro for the biofilm production. A similar study showed a $59.0 \%$ biofilm producers and $41 \%$ biofilm non producers ${ }^{18}$. Many other studies reported biofilm in UPEC was $13.5 \%, 16.0 \%$ and $6.0 \%{ }^{5,25,26}$. Biofilm production was found to be highly prevalent in UTIs patients. The routine culture \& sensitivity should be done along with the testing of biofilm production.

Among 106 UPEC, 38 (35.85\%) were ESBL producers of which $89.47 \%$ were biofilm positive and $10.53 \%$ were biofilm negative. $64.15 \%$ were non ESBL producers of which $64.71 \%$ were biofilm positive and $35.29 \%$ were biofilm negative. The biofilm forming ability was found to be significantly high in ESBL +ve strains of UPEC than that of ESBL negative strains. This is in accordance with other researchers ${ }^{5,24,25,27-31}$.

In this study, the antibiotic resistance of biofilm producing UPEC was found significantly higher as compare to nonbiofilm producing UPEC. Microorganisms growing in a biofilm are intrinsically resistant to many antibiotics increasing the antibiotic resistance upto 1000 folds and high antimicrobial concentrations are required to inactivate organisms growing in a biofilm ${ }^{32,33}$. This may be because of insufficient concentration of the antibiotics reaching some areas of the biofilm and metabolic inactiveness of the bacteria located at the base of the biofilms ${ }^{34}$. It was documented in the previous research that recurrent UTIs in the women were commonly due to the biofilm producing UPEC strains. Therefore, the study of factors contributing to biofilm formation is important to reveal the new therapeutics agents, which can be useful for the treatment of recurrent and chronic UTIs. ${ }^{35}$

The prevalence of MDR in biofilm producers has been reported more in comparison to biofilm negative strains ${ }^{6}$. The resistance to multiple drugs could be due to spread of hsopaitalzed UPEC strains and the high prevalence of ESBL positive isolates is possibly due to the creation of selective drug pressure because of common use of cephalosporins and other antimicrobials in our study area.

In the current study, among oral antibiotics, nitrofurantoin was $72.0 \%$ sensitive in biofilm producers and $88.0 \%$ sensitive in ESBL \& Biofilm producers where as in the other study done at Rohtak, India Nitrofurantoin was $100 \%$ effective in both cases ${ }^{5}$. Fluoroquinolones were found to be more resistant in biofilm producing isolates (Levofloxacin: 95.0\%; Ciprofloxacin: 97.0\%). In the other similar studies, $75.0 \%$ resistance was found in Ciprofloxacin, $68.0 \%$ in ofloxacin, $75.0 \%$ in norfloxacin ${ }^{31}$ and $13.0 \%$ resistance in Levofloxacin, $41.0 \%$ in Norfloxacin ${ }^{18}, 70.0 \%$ - Ciprofloxacin, $78.0 \%$ - Norfloxacin ${ }^{36}$. Nitrofurantoin was the only oral drug which was shown good sensitivity in Biofilm positive and ESBL producing UPEC, and this drug can be reserved drug for the treatment of UTIs in hospitalized patients. Knowledge of the nature of biofilm in UTIs will help in more effective treatment guidelines.

This study revealed the resistant rates of $27.0 \%$ and $63.0 \%$ to the aminoglycosides, Amikacin and Gentamicin by biofilm producers. $70.0 \%$ and $86.0 \%$ resistance was 
reported by Poovendran et $a l^{25}, 0.0 \%$ and $20.8 \%$ resistance was reported by Shahidul et $a l^{20}, 46.0 \%$ and $36.0 \%$ resistance was observed by Atray et $\mathrm{al}^{36}$.

Increasing irrational and haphazard use of antibiotics, sales of substandard antibiotics and transmission of drug resistant bacteria among people may be responsible for the rise in antibiotic resistance among the bacteria ${ }^{37}$. Antimicrobial resistance has become a serious global public health issue. Infections caused by drug resistant bacteria are responsible for increased morbidity and ${ }^{38}$. The selection of the antibiotics for treatment of the bacterial infections should be based on culture and sensitivity reports.

\section{Acknowledgment}

The study was carried out at Bacteriology section, Department of Microbiology, Modern Diagnostic and Research Centre, Gurugram

\section{References}

1. Das, R. N. et al., Frequency and susceptibility profile of pathogens causing urinary tract infections at a tertiary care hospital in western Nepal. Singapore Med. J. 47, 281-5 (2006).

2. Johnson, J. R. Virulence factors in Escherichia coli urinary tract infection. Clin. Microbiol. Rev. 4, 80-128 (1991).

3. Potera, C. Forging a link between biofilms and disease. Science 283, 1837, 1839 (1999).

4. Sahly, H. et al., Extended-spectrum beta-lactamase production is associated with an increase in cell invasion and expression of fimbrial adhesins in Klebsiella pneumoniae. Antimicrob. Agents Chemother. 52, 3029-34 (2008).

5. Mittal, S., Sharma, M. \& Chaudhary, U. Biofilm and multidrug resistance in uropathogenic Escherichia coli. Pathog.
Glob. Health 109, 26-9 (2015).

6. Costerton, J. W., Stewart, P. S. \& Greenberg, E. P. Bacterial biofilms: a common cause of persistent infections. Science 284, 1318-22 (1999).

7. Gupta, K., Hooton, T. M. \& Stamm, W. E. Increasing antimicrobial resistance and the management of uncomplicated community-acquired urinary tract infections. Ann. Intern. Med. 135, 4150 (2001).

8. Costerton, J. W., Lewandowski, Z., Caldwell, D. E., Korber, D. R. \& Lappin-Scott, H. M. Microbial Biofilms. Annu. Rev. Microbiol. 49, 711-745 (1995).

9. Costerton, J. W. et al., Bacterial Biofilms in Nature and Disease. Annu. Rev. Microbiol. 41, 435-464 (1987).

10. Nickel, J. C., Ruseska, I., Wright, J. B. \& Costerton, J. W. Tobramycin resistance of Pseudomonas aeruginosa cells growing as a biofilm on urinary catheter material. Antimicrob. Agents Chemother. 27, 619-24 (1985).

11. O'Toole, G., Kaplan, H. B. \& Kolter, R. Biofilm Formation as Microbial Development. Annu. Rev. Microbiol. 54, 49-79 (2000).

12. CLSI. Performance Standards for Antimicrobial Susceptibility Testing; Nineteenth Informational Supplement. CLSI document M100-S19. Wayne, PA. USA. 29, (2009).

13. Christensen, G. D., Simpson, W. A., Bisno, A. L. \& Beachey, E. H. Adherence of slime-producing strains of Staphylococcus epidermidis to smooth surfaces. Infect. Immun. 37, 318-26 (1982).

14. Freeman, D. J., Falkiner, F. R. \& Keane, C. T. New method for detecting slime production by coagulase negative staphylococci. J. Clin. Pathol. 42, 8724 (1989).

15. Christensen, G. D. et al., Adherence of 
coagulase-negative staphylococci to plastic tissue culture plates: a quantitative model for the adherence of staphylococci to medical devices. $J$. Clin. Microbiol. 22, 996-1006 (1985).

16. STEPANOVIĆ, S. et al., Quantification of biofilm in microtiter plates: overview of testing conditions and practical recommendations for assessment of biofilm production by staphylococci. $J$. Pathol. Microbiol. Immunol. 115, 891899 (2007).

17. Naik, J. \& Desai, P. Antibiotic resistance pattern in urinary isolates of Escherichia coli with special reference to extended spectrum $\beta$-Lactamases production. Int. J. Pharm. Life Sci. 3, 1498-1502 (2012).

18. Poovendran, P. \& Ramanathan, N. In Vitro Study On Antibiotic Susceptibility Pattern Of Biofilm Producing Uropathogenic Escherichia Coli Isolates And Their Molecular Characterization. Asian J. Pharm. Clin. Res. 7, 181-185 (2014).

19. S. NIVEDITHA, S. PRAMODHINI, S. UMADEVI, SHAILESH KUMAR, S. $\mathrm{S}$. The Isolation and the Biofilm Formation of Uropathogens in the Patients with Catheter Associated Urinary Tract Infections (UTIs). J. Clin. Diagnostic Res. 6, 1478-1482 (2012).

20. Shahidul, K. M., Asma, A., Farahnaaz, F. \& Sunjukta, A. Determination of Antibiotic resistance pattern of Biofilm producing Pathogenic Bacteria associated with UTI. Int. J. Drug Dev. Res. 5, 312-319 (2013).

21. Soto, S. M. et al., Implication of biofilm formation in the persistence of urinary tract infection caused by uropathogenic Escherichia coli. Clin. Microbiol. Infect. 12, 1034-1036 (2006).

22. Madigan, E. \& Neff, D. F. Care of patients with long-term indwelling urinary catheters. Online J. Issues Nurs.
8, 7 (2003).

23. Hassan, A. et al., Evaluation of different detection methods of biofilm formation in the clinical isolates. Brazilian $J$. Infect. Dis. - Elsevier 15, 305-311 (2011).

24. Hittinahalli, V., Karjigi, S. K. \& Mallika Reddy, K. Correlation Between Biofilm Formation Of Uropathogenic Escherichia Coli And Its Antibiotic Resistance Pattern. J. Evol. Med. Dent. Sci.

25. Ponnusamy, P., Natarajan, V. \& Sevanan, M. In vitro biofilm formation by uropathogenic Escherichia coli and their antimicrobial susceptibility pattern. Asian Pac. J. Trop. Med. 5, 210-213 (2012).

26. Murugan, S., Devi, P. U. \& John, P. N. Antimicrobial Susceptibility Pattern of Biofilm Producing Escherichia coli of Urinary Tract Infections. Curr. Res. Bacteriol. 4, 73-80 (2011).

27. Neupane, S. et al., Correlation between biofilm formation and resistance toward different commonly used antibiotics along with extended spectrum beta lactamase production in uropathogenic Escherichia coli isolated from the patients suspected of urinary tract infections visit. Antimicrob. Resist. Infect. Control 5, 5 (2016).

28. Anandkumar, H., Soham, G., Vinodkumar, C., Rao, A. \& Srinivasa, H. Detection of Cell Surface Hydrophobicity and Biofilm formation among ESBL-and non-ESBL-producing uropathogenic Escherichia coli. J. Med. Educ. Res. 2,

29. Nair, B. T., Bhat, K. G. \& Shantaram, M. Invitro biofilm production and virulence factors of uropathogenic Escherichia coli. Int J Pharm Bio Sci 4, 951-956 (2013).

30. Subramanian, P., Kumar, S. \& Stephen, S. Determination of correlation between 
biofilm and extended spectrum $\beta$ lactamases producers of Enterobacteriaceae. Sch. Res. J. 2,

31. Tadepalli, S., Prudhivi, S., Myneni, R. B. \& Rao, S. Biofilm formation in uropathogenic Escherichia coli isolates and its association with extended spectrum betalactamase production and drug resistance. Saudi J. Pathol. Microbiol. 1, 60-64 (2016).

32. Mah, T. F. \& O'Toole, G. A. Mechanisms of biofilm resistance to antimicrobial agents. Trends Microbiol. 9, 34-9 (2001).

33. Stewart, P. S. \& Costerton, J. W. Antibiotic resistance of bacteria in biofilms. Lancet (London, England) 358, 135-8 (2001).

34. Soto, S. M. Importance of Biofilms in Urinary Tract Infections: New Therapeutic Approaches. Adv. Biol. 2014, 1-13 (2014).

35. Warren, J. W. Catheter-associated urinary tract infections. Int. J. Antimicrob. Agents 17, 299-303 (2001).

36. Atray, D. \& Atray, M. Correlation between Biofilm Production and Antibiotic Resistance Pattern in Uropathogenic Escherichia coli in Tertiary Care Hospital in Southern Rajasthan, India. Int.J.Curr.Microbiol.App.Sci 4, 640646 (2015).

37. Gautam, R. et al., Antimicrobial Susceptibility Patterns of Escherichia Coli From Various Clinical Sources. $J$. Chitwan Med. Coll. 3, 14-17 (2013).

38. Awasthi, T. R., Dutt Pant, N. \& Dahal, R. Prevalence of Multidrug Resistant Bacteria in Causing Community Acquired Urinary Tract Infection Among the Patients Attending Outpatient Department of Seti Zonal Hospital, Dhangadi, Nepal. Nepal J. Biotechnol. 3, 55-59

\section{How to cite this article:}

Rishabh Rajput, Surendra Sarsaiya, Naresh Kumar and Swati Shekhawat. 2021. Biofilm Formation in UPEC Isolates and its Association with Extended-Spectrum $\beta$-Lactamase Production and MDR in Hospitalized Patients: Gurugram, India. Int.J.Curr.Microbiol.App.Sci. 10(02): 673-685. doi: https://doi.org/10.20546/ijcmas.2021.1002.081 